'S Vedovato, ${ }^{2} \mathrm{~S}$ Visentin, ${ }^{2} \mathrm{E}$ Cosmi, ${ }^{3} \mathrm{~F}$ Cavallin, ${ }^{4} \mathrm{M}$ Mion, ${ }^{4} \mathrm{M}$ Zaninotto, ${ }^{3} \mathrm{D}$ Trevisanuto, ${ }^{3} \mathrm{~V}$ Zanardo. ${ }^{1}$ Neonatal Intensive Care Unit, Department of Paediatrics, Hospital San Bortolo of Vicenza, Vicenza; ${ }^{2}$ Maternal Fetal Medicine Unit, Department of Woman and Child Health; ${ }^{3}$ Department of Pediatrics, University of Padua School of Medicine; ${ }^{4}$ Department of Laboratory Medicine, University-Hospital of Padova, Padova, Italy

Background and Aims Intrauterine growth restriction (IUGR) complicated by umbilical artery vasculopathy may have adverse effects on integrity and function of vascular endothelium and has been associated to atherosclerosis and to glomerulosclerosis risk in adulthood. Aortic intima media thickening, a preclinical sign of atherosclerosis, has been documented from intrauterine life, whereas the first signs of glomerulosclerosis have not been determined. So the aim of this study was to compare albuminuria/creatiniruria ratio (ACR) in IUGR and appropriate for gestational age (AGA) neonates.

Methods A prospective cohort study has been performed on 25 IUGR consecutive fetuses evaluated at Department of Woman and Child Health of Padua University between December 2009 and December 2010. They were considered IUGR if the estimated fetal weight (EFW) was below 10th percentile and the umbilical artery pulsatility index (PI) $>2$ standard deviations. Each IUGR newborn was matched with 2 controls AGA neonates, of the same gestational age \pm 1 week. They were considered AGA if the EFW was between 10th and 90th percentile. A urine sample was collected at 24-72 hours after birth for ACR determination.

Results Among the 25 IUGR fetuses enrolled, 2 were excluded because 1 had trisomy 21 and 1 renal agenesis. The remaining 23 were matched with 46 AGA newborns. ACR was significantly higher in IUGR compared to AGA newborns: median (IOR) 183.0 (113.6-264.7) vs 122.8 (72.5-191.9); $\mathrm{p}=0.04$.

Conclusions IUGR is associated with significantly greater albuminuria at birth. This may be an early marker of glomerulosclerosis, which leads to renal disease in adulthood.

\section{SENSORINEURAL HEARING LOSS AFTER NORMAL NEONATAL HEARING SCREENING IN VERY PRETERM INFANTS}

doi:10.1136/archdischild-2012-302724.1217

I van Noort-van der Spek, A Goedegebure, N Weisglas-Kuperus. Erasmus MC - Sophia Children's Hospital, Rotterdam, The Netherlands

Background and Aim To determine the prevalence of sensorineural hearing loss (SNHL) measured by auditory brainstem response $(\mathrm{ABR})$ at 2 years of corrected age in very preterm (VPT) infants with normal neonatal hearing screening (NHS).

Methods 85 VPT infants (gestational age $<32$ weeks, birth weight $<1850$ grams), born between October 2008 and February 2010, underwent NHS by automated auditory brainstem response (AABR) shortly after birth and $A B R$ at 2 years of corrected age. The frequency of SNHL in VPT infants with bilateral pass AABR was analyzed. SNHL was estimated from the ABR responses and defined as a hearing loss $>20 \mathrm{~dB}$ for at least one ear, after correction for possible conductive hearing loss. Univariate analyses were performed to identify risk factors associated with SNHL.

Results Bilateral pass AABR shortly after birth was found in 81 (95\%) VPT infants. In spite of bilateral pass AABR, 23 (29\%) out of 78 VPT infants had hearing loss, defined as an estimated hearing loss $>20 \mathrm{~dB}$ HL for at least one ear, measured by $\mathrm{ABR}$ at 2 years of corrected age. Of these VPT infants, $5(6 \%)$ had SNHL. Proven sepsis was found in $4(80 \%)$ VPT infants with SNHL compared to 23 $(32 \%)$ VPT infants without SNHL $(\mathrm{P}<0.05)$.

Conclusions SNHL could be identified in 6\% of 2-year-old VPT infants in spite of normal NHS shortly after birth. Follow-up audiometric testing after normal NHS is recommended for VPT infants who had proven sepsis in the neonatal period.

\section{INCIDENCE, MORTALITY, MORBIDITY AND COSTS OF LATE PRETERM BIRTHS}

doi:10.1136/archdischild-2012-302724.1218

${ }^{1} \mathrm{C}$ Bulut, 'T Gürsoy, ${ }^{2} \mathrm{~F}$ Ovalı. 'Zeynep Kamil Maternity and Childrens' Disease Training and Research State Hospital; 'Zeynep Kamil Maternity and Childrens' Disease Training and Research State Hospital, Istanbul, Turkey

Background and Aims Since 2005, babies born between 34 0/7 weeks and 36 6/7 weeks are defined as late preterm babies. We aimed to investigate the rates of mortality and morbidity of late preterm babies born at our hospital and compare them with babies born atterm.

Material and Methods A retrospective chart analysis was performed on babies born at our hospital between 2006 and 2008 Demographic features, complications and mortalities were recorded. Results During 3 years, 41752 babies were delivered in our hospital $6742(16.2 \%)$ of them were late preterm and $30055(71.9 \%)$ were term babies. The rate of hospitalizations ( $6.5 \%$ vs $17.5 \%)$, mortality ( $3.6 \%$ vs. $5 \%$ ) and duration of hospitalizations (4 days vs 7 days mean) were significantly higher in the late preterm group. $66 \%$ of late preterm infants were transferred to other units for various reasons. When stratified according to gestational age, there were no significant differences in terms of mortality and morbidity among the subgroups. However, the duration of hospitalizations and the costs of babies born at 34 weeks were significantly higher than those of other babies.

Conclusion Late preterm babies have increased rates of mortality and morbidity, including higher costs, hence constitute an important risk group, which need to be followed up regularly. The mortality and morbidity rates did not appear to increase with decreasing gestational age in late preterm babies but the duration of hospitalization and the costs were higher in babies born at 34 weeks.

\section{DIFFERENCES IN MORTALITY/MORBIDITY WITH A COMPLETE COURSE OF ANTENATAL STEROIDS COMPARED TO AN INCOMPLETE/NO COURSE IN EXTREMELY PREMATURE NEONATES}

doi:10.1136/archdischild-2012-302724.1219

'D Wong, 1,2ME Abdel-Latif, ${ }^{1,2} \mathrm{AL}$ Kent. ${ }^{1}$ Australian National University, Medical School; ${ }^{2}$ Dept of Neonatology, Canberra Hospital, Canberra, ACT, Australia

Background Antenatal steroids have been shown to reduce mortality and morbidity in neonates born less than 29 weeks gestation. Counselling of parents regarding outcomes is generally based on data that includes all neonates whether they have received a complete course of antenatal steroids or not. In the acute setting where delivery is imminent more accurate estimation of outcomes for parents is required.

Aims To determine the differences in survival, short and long term morbidity for those neonates receiving no antenatal steroids or an incomplete course of steroids in comparison to those receiving a complete course.

Methods A retrospective review of prospectively collected data from 10 NICU'S between January 1998 to December 2004

Results 2549 neonates were included in the study with 319 $(12.5 \%)$ not given any ante-natal steroids. Hospital mortality was significantly worse without steroids $(30 \%$ versus $20 \% \mathrm{p}<0.001)$. Those with no steroid coverage were more likely to have NEC $(11 \%$ vs $7 \%$; $p=0.018)$ and Grade 3 or 4 IVH ( $19 \%$ vs $12 \%$; $p=0.001)$. In a multivariate model, factors predictive of mortality included: lack of antenatal steroids, male gender, smaller gestation and hypertensive disease of pregnancy.

Long-term data was available for 1473 survivors. There was no difference in long term neurological outcome between those not receiving steroids and those receiving any steroids. 